\title{
The CUORE Fast Cooling System
}

\section{Carmine Elvezio Pagliarone ${ }^{1}$}

Department of Civil and Mechanical Engineering (DICeM), University of Cassino and Southern Lazio (UNICLAM), Via G. Di Biasio, 03043 Cassino (FR), Italy

Laboratori Nazionali del Gran Sasso, Via G. Acitelli, 22, 67100 Assergi (AQ), Italy

E-mail: pagliaronedunicas.it

\section{Luigi Cappelli}

Department of Physics, University of California, 366 LeConte Hall MC 7300, Berkeley, CA,USA

Laboratori Nazionali del Gran Sasso, Via G. Acitelli, 22, 67100 Assergi (AQ), Italy

\section{Carlo Bucci, Paolo Gorla}

Laboratori Nazionali del Gran Sasso, Via G. Acitelli, 22, 67100 Assergi (AQ), Italy

\section{Damiano D‘Aguanno, Fabrizio Marignetti}

Department of Electrical and Information Engineering (DIEI), University of Cassino and Southern Lazio (UNICLAM), Via G. Di Biasio, 03043 Cassino (FR), Italy

\section{Gianni Erme}

Department of Civil and Mechanical Engineering (DICeM), University of Cassino and Southern Lazio (UNICLAM), Via G. Di Biasio, 03043 Cassino (FR), Italy

\section{Sehban Kartal}

Department of Physics, High Energy and Plasma Division, Istanbul University, 34134 VeznecilerIstanbul, Turkey

\begin{abstract}
The Cryogenic Underground Observatory for Rare Events (CUORE) is a Ton-scale Neutrino-less Double Beta Decay experiment that uses $998 \mathrm{TeO}_{2}$ crystal bolometers that need to be operated at a baseline temperature of around $10 \mathrm{mK}$. The large volume and masses involved require a dedicated precooling system that allows the apparatus to reach a threshold temperature after which the Dilution Unit, a mK-scale refrigerator, can start the final cool-down. The CUORE Fast Cooling System (FCS) has been developed, constructed and recently used for the CUORE precooling stage. It mainly consists of a cryostat with heat exchangers that use 3 GiffordMcMahon refrigerators, a He blower, a filtering module and several sensors that allow to monitor and control the system during this very delicate cooldown procedure. The present work describes the FCS and summarize the FCS performances during the first full CUORE detector cooldown.
\end{abstract}

The European Physical Society Conference on High Energy Physics

5-12 July, 2017

Venice

\footnotetext{
${ }^{1}$ Speaker

(C) Copyright owned by the author(s) under the terms of the Creative Commons 


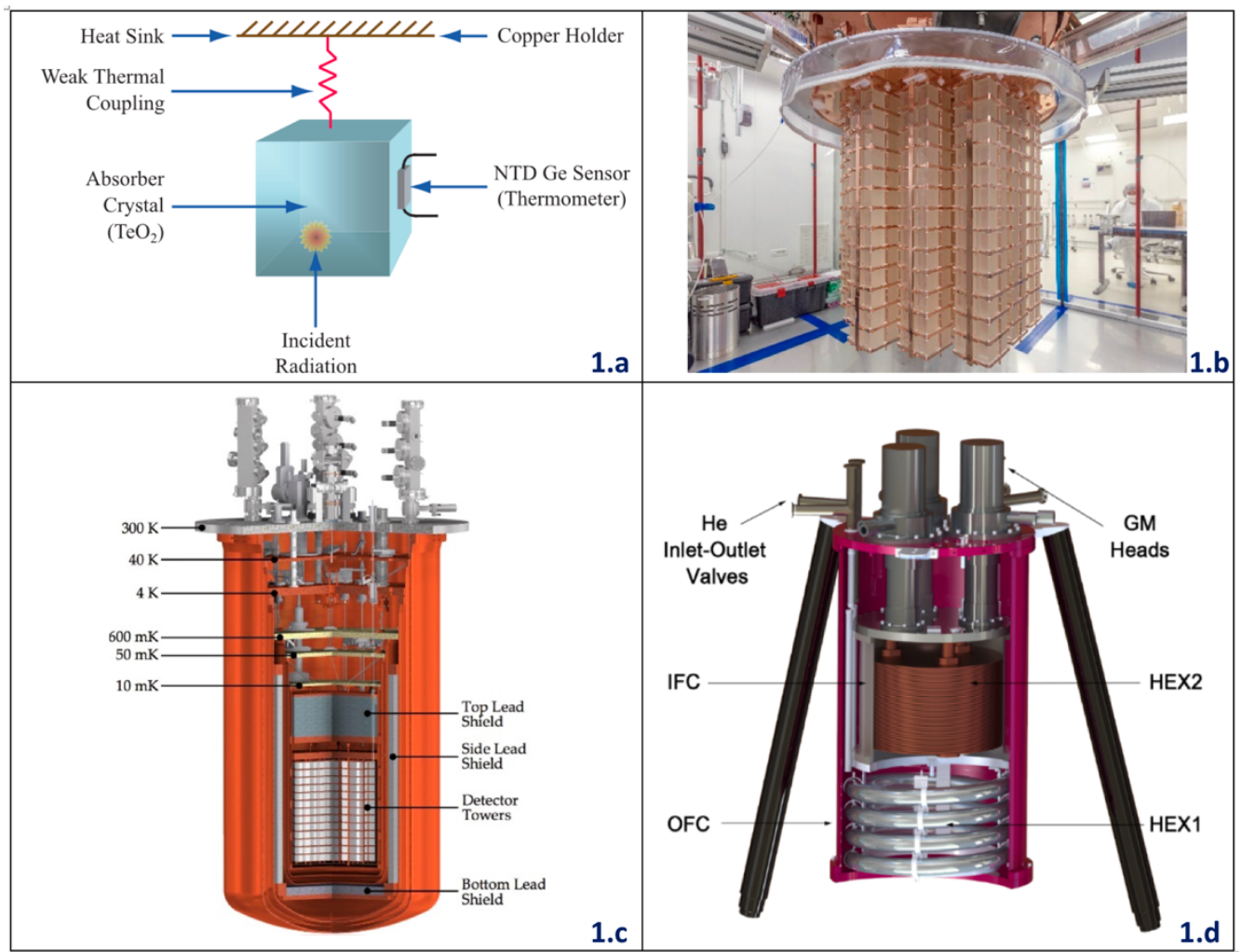

Figure 1: a) CUORE $\mathrm{TeO}_{2}$ bolometer scheme; b) the 19 CUORE Towers during the assembling; c) Layout of CUORE Experiment; d) the Fast Cooling Unit (FCU).

\section{Introduction}

The Cryogenic Underground Observatory for Rare Events (CUORE) is an experiment, located in the Gran Sasso National Laboratory (LNGS) of the National Institute for Nuclear Physics (INFN) in Italy, presently taking data to look for Neutrino-less Double Beta Decay (0v2 $\beta$ ) and Dark Matter (DM) [1]. In order to search for these rare events, a very low radioactive contamination and very low temperatures are required $(\sim 10 \mathrm{mK})$. For these reasons CUORE is located in an underground laboratory and its detector is kept at a stable temperature of about 10 $\mathrm{mK}$. The CUORE detector consists of 988 ultra radiopure $\mathrm{TeO}_{2}$ crystals (see Fig. 1.a) arranged in 19 identical towers as shown in Fig. 1.b. Each crystal acts as a bolometer (i.e. an absorber that is connected to a heat sink, a body of constant temperature, through a thermal coupling as shown in Fig. 1.a and Fig. 1.b). To bring the detector at such a low temperature, a challenging cryostat has been design to host the detector as shown in Fig. 1.c. The cryostat is composed by 6 coaxial vessels, kept at $300 \mathrm{~K}, 40 \mathrm{~K}, 4 \mathrm{~K}, 600 \mathrm{mK}, 50 \mathrm{mK}$ and $10 \mathrm{mK}$ respectively [2]. The $300 \mathrm{~K}$ and 4 $\mathrm{K}$ vessels are vacuum tight, and the detector itself is placed inside the innermost one. Several lead shields are located on top, bottom and lateral side of the detector to prevent radioactivity contamination from external vessels (ancient Roman lead has been used). The total CUORE mass to be cooled down is $17209 \mathrm{~kg}$. Given such a huge mass, the cooling system plays a fundamental role in order to obtain the required temperature in a suitable time. The CUORE cryostat is equipped with two cooling systems: Pulse Tubes (PTs) and a Dilution Refrigerator (DR). The PTs keep the detector down to $4 \mathrm{~K}$ and the DR down to $10 \mathrm{mK}$. In order to have a faster cooling procedure, a pre-cooling phase has been developed in which an external system, the Fast Cooling System, cools down the detector to a temperature of tens of $\mathrm{K}$ in a shorter time. 


\section{The CUORE Fast Cooling System}

The CUORE Fast Cooling System consists of cryogen free coolers (five PTs) and an external system that flows cold ${ }^{4} \mathrm{He}$ inside the CUORE Inner Vacuum Chamber (IVC). This external system involves a Fast Cooling Unit (FCU), it contains two heat exchangers, a gas compressor, double-walled flexible pipes, a filtering system and several other components and sensors. The FCU is cooled by 3 Gifford-McMahon Cryocoolers and it is depicted in Fig. 1.d. It has been manufactured by Leiden Cryogenics ${ }^{\mathrm{TM}}$, The Netherlands. The two internal heat exchangers are made one of Oxygen-Free High Conductivity copper (OFHC-Cu) and the other of double pipe bellows; they are referred to as HEX2 and HEX1, in Fig. 1.d. The ${ }^{4} \mathrm{He}$ gas, taken from a liquid helium dewar, is filtered and blows to the FCU to be cooled; thus, it enters the CUORE cryostat, exchanges heat and then is taken out to the FCU to start again the circulation. The entire precooling process is critical since several requirements need to be fulfilled; in order to avoid damages to the crystals the temperature gradient needs to be $\Delta \mathrm{T}<40 \mathrm{~K}$, the pressure has to be within a specific range $\left(\Delta \mathrm{P}=\mathrm{P}_{\text {out }}-\mathrm{P}_{\text {in }}<350 \mathrm{mbar}\right)$ and the He flux cannot exceed $5 \mathrm{~g} / \mathrm{s}$. Thus, a Fast Cooling Monitor and Control System (FCMS) has been developed in order to have the precooling process under control. Thanks to several sensors, both automatic and manual actions can be taken (i.e. refill of $\mathrm{He}$ in the circuit, changing compressor frequency, open valves, setup of optimized speed and so on). Big effort has been put to develop both hardware and software safety systems, together with the related alarms. The high-level interfaces are based on Labview ${ }^{\circledR}$.

\subsection{FCS Performance \& Results}

After several months of commissioning and tests, the FCS has been used to cool down the CUORE cryostat initially without the detector (installed in summer 2016) and then with it, to start data taking [3]. In both cases, the FCS brought the cryostat from room temperature down to $50 \mathrm{~K}$ and $100 \mathrm{~K}$ respectively, in about 10 days. Even if the FCS is able to reach lower temperatures it has been switched off prematurely in order to perform tests on PTs. During the last run, a peak value of $(1.09 \pm 0.02) \mathrm{K} / \mathrm{h}$ has been reached while an average cooling speed of approximately $0.70 \mathrm{~K} / \mathrm{h}$ has been kept during all the pre-cooling phase. Given the results, CUORE pre-cooling performances can be considered very good. Further improvements are foreseen.

\section{Conclusions}

The Fast Cooling System has been designed and operated to speed up the pre-cooling phase of CUORE experiment. Around 20 tons of mainly copper and lead have been cooled down to less than $100 \mathrm{~K}$ in only 10 days. The mean cooling speed was approximately $0.70 \mathrm{~K} / \mathrm{h}$. The entire precooling process has been supervised by a custom-made Fast Cooling Monitor and Control System which allowed to match the design and safety specifications. The resulting cooling performances can be considered very good, while future improvements are foreseen.

\section{References}

[1] D. Santone et al., The CUORE cryostat and its bolometric detector, JINST 12 (2017) no.02, C02055.

[2] C. Ligi et al., The CUORE Cryostat: A 1-Ton Scale Setup for Bolometric Detectors, J.Low.Temp.Phys. 184 (2016) no.3-4, 590-596.

[3] L. Cappelli, et al., Pre-cooling of ton-scale particle detectors in low radioactivity environments, in proceedings of $28^{\text {th }}$ International Conference on Low Temperature Physics, Aug 2017, Sweden. 